\title{
Pathological analysis and surgical modalities selection of cT1NOMO solitary papillary thyroid carcinoma in the isthmus
}

\author{
Li-Zhuo Zhang ${ }^{1,2,3} \wedge$, Jia-Jie $\mathrm{Xu}^{2,3 \#}$, Xin-Yang Ge ${ }^{4}$, Ke-Jing Wang ${ }^{5}$, Zhuo Tan ${ }^{2,3}$, Tie-Feng Jin ${ }^{1,2,3}$, \\ Wan-Chen Zhang ${ }^{1,2,3}$, Qing-Lin Li ${ }^{6}$, Ding-Cun Luo ${ }^{7}$, Ming-Hua Ge ${ }^{2,3}$
}

${ }^{1}$ Second Clinical Medical College, Zhejiang Chinese Medical University, Hangzhou, China; ${ }^{2}$ Department of Head and Neck Surgery, Center of Otolaryngology-Head and Neck Surgery, Zhejiang Provincial People's Hospital, People's Hospital of Hangzhou Medical College, Hangzhou, China; ${ }^{3}$ Key Laboratory of Endocrine Gland Diseases of Zhejiang Province, Zhejiang Provincial People's Hospital, People's Hospital of Hangzhou Medical College, Hangzhou, China; ${ }^{4}$ College of Letters and Science, University of California, Los Angeles, Los Angeles, California, USA; ${ }^{5}$ Department of Head and Neck Surgery, Cancer Hospital of University of Chinese Academy of Sciences (Zhejiang Cancer Hospital), Hangzhou, China; ${ }^{6}$ Department of Scientific Research, Cancer Hospital, University of Chinese Academy of Sciences, Hangzhou, China; ${ }^{7}$ Department of Surgical Oncology, Affiliated Hangzhou First People's Hospital, Zhejiang University School of Medicine, Hangzhou, China

Contributions: (I) Conception and design: MH Ge, JJ Xu, LZ Zhang; (II) Administrative support: MH Ge, QL Li, DC Luo; (III) Provision of study materials or patients: KJ Wang, DC Luo, Z Tan; (IV) Collection and assembly of data: XY Ge, WC Zhang, LZ Zhang; (V) Data analysis and interpretation: LZ Zhang, TF Jin; (VI) Manuscript writing: All authors; (VII) Final approval of manuscript: All authors.

\#These authors contributed equally to this work.

Correspondence to: Ming-Hua Ge. Department of Head and Neck Surgery, Center of Otolaryngology-Head and Neck Surgery, Zhejiang Provincial People's Hospital, People's Hospital of Hangzhou Medical College, 158 Shangtang Road, Xiacheng District, Hangzhou 310014, China. Email: gemingh@163.com; Qing-Lin Li. Department of Scientific Research, Cancer Hospital, University of Chinese Academy of Sciences, 1 Banshan East Road, Hangzhou 310022, China. Email: qinglin200886@126.com; Ding-Cun Luo. Department of Surgical Oncology, Affiliated Hangzhou First People's Hospital, Zhejiang University School of Medicine, 261 Huansha Road, Shangcheng District, Hangzhou 310006, China. Email: 1dc65@163.com.

Backgroundk prognosis, identify clinicopathological characteristics, and determine optimal modalities for cT1N0M0 solitary papillary thyroid carcinoma in the isthmus (PTCI).

Methods: The clinical data of 124 patients with cT1N0M0 solitary PTCI from 3 medical centers were analyzed retrospectively. Of these, 32 participants had undergone total thyroidectomy plus unilateral central neck dissection, 36 had received total thyroidectomy plus bilateral central neck dissection, 24 had lessthan-total thyroidectomy plus unilateral central neck dissection, and 32 had less-than-total thyroidectomy plus bilateral central neck dissection. We compared the effects of different surgical modalities and clinicopathological characteristics on the prognosis of cT1N0M0 solitary PTCI.

Results: There was no significant difference in postoperative recurrence-free survival between participants who received different extents of central region lymph node dissection and thyroidectomies $(\mathrm{P}>0.05)$. Temporary hypocalcemia occurred in participants who underwent total thyroidectomy plus bilateral central neck dissection [chi-square $\left(\chi^{2}\right)=7.87, \mathrm{P}=0.005$ ]. Tumors with primary lesions $\geq 0.55 \mathrm{~cm}$ were prone to have central lymph node metastasis [95\% confidence interval (CI): 0.51 to $0.71, \mathrm{P}=0.047$ ]. Multiple logistic analysis suggested that age over 55 years [odds ratio $(\mathrm{OR})=11.90,95 \%$ CI: 1.36 to $104.03, \mathrm{P}=0.025$ ], tumor size greater than $0.55 \mathrm{~cm}(\mathrm{OR}=4.16,95 \% \mathrm{CI}: 1.28$ to $13.52, \mathrm{P}=0.018)$, and absence of nodular goiter (OR $=2.57,95 \% \mathrm{CI}: 1.05$ to $6.32, \mathrm{P}=0.04)$ were risk factors for central lymph node metastasis of patients with cT1N0M0 solitary PTCI.

Conclusions: Less-than-total thyroidectomy is recommended for patients with cT1N0M0 solitary PTCI. Central lymph node dissection is recommended for patients who are prone to have central occult lymph node metastases with tumor size $\geq 55 \mathrm{~cm}$, older than 55 years, and without nodular goiter.

\footnotetext{
^ ORCID: 0000-0002-4460-1743.
} 
Keywords: Papillary thyroid carcinoma (PTC); thyroid isthmus; lymph node metastases

Submitted May 10, 2021. Accepted for publication Aug 05, 2021.

doi: $10.21037 /$ gs-21-357

View this article at: https://dx.doi.org/10.21037/gs-21-357

\section{Introduction}

Thyroid cancer is the most common type of malignant tumor of the endocrine system and has seen a persistently increasing trend in incidence during recent years (1). The most common pathological type of thyroid cancer is papillary thyroid carcinoma (PTC), estimated to comprise $85 \%$ of diagnosed thyroid cancer cases (2). The recent increase in thyroid cancer can be attributed to a rapid increase in PTC (3). At present, the main clinical treatment for PTC is lobectomy plus isthmusectomy with central lymph node dissection, while total thyroidectomy with $\mathrm{I}^{131}$ radiotherapy is recommended for patients with highrisk factors such as multiple tumors, capsular invasion, and lymph node metastasis. The malignant degree of PTC is lower than that of other solid tumors, and the 10-year survival rate of PTC patients has reached up to over $90 \%$ after the provision of formal care (4).

The isthmus of the thyroid is a narrow banded structure connecting the left and right lobes of the thyroid gland, located directly in front of the trachea with its forepart covered by pretracheal fascia, strap muscles, and anterior neck skin. Given the small volume and secluded location of the isthmus, the incidence of papillary thyroid carcinoma in the isthmus (PTCI) is low, and there have been few relevant reports to date. The extent of PTCI surgical resection has not been specified by authoritative associations such as the American Thyroid Association (ATA) and the European Thyroid Association (ETA) $(5,6)$. Some researchers have found that the pathological features of PTCI are multifocal, it can easily invade capsular and surrounding issues (7-13), and total thyroidectomy is recommended to address this. However, reports have found no significant difference in prognosis between patients with micropapillary PTCI treated with partial thyroidectomy and those treated with total thyroidectomy (14). Moreover, whether patients with cT1N0M0 solitary PTCI should receive central lymph node dissection, or unilateral or bilateral central lymph node dissection has not been fully elucidated.

Following the improvement of resolution for ultrasound used in screening, there has been a trend of early and increased discovery for thyroid cancer, including PTCI (15). The lack of clarity regarding the optimal treatment approach has the unfortunate effect of delaying timely intervention. Therefore, our study aimed to analyze clinicopathological characteristics and the effect of different surgical modalities on the prognosis of patients and explore the appropriate surgical extent for c'T1N0M0 solitary PTCI. We present the following article in accordance with the STARD reporting checklist (available at https://dx.doi. org/10.21037/gs-21-357).

\section{Methods}

\section{Participant cohort}

We collected a total of 18,550 medical records of PTC patients who had undergone surgery for the first time at Zhejiang Provincial People's Hospital, Zhejiang Cancer Hospital, and Hangzhou First People's Hospital from January 2001 to December 2017. By analyzing the results of preoperative color ultrasound examination of the thyroid and cervical lymph nodes, 356 patients with isthmic cancer were selected, including 124 patients with cT1N0M0 solitary PTCI. Patients with multiple cancers, extrathyroidal extension, tumors larger than $2 \mathrm{~cm}$ in diameter, family history of thyroid cancer, previous radiation exposure, postoperative radioiodine therapy, and suspected cervical lymph node metastasis or distant metastasis were excluded. Ultrasound imaging characteristics and pathological characteristics of all participants were analyzed, including age, gender, tumor size, presence of capsular invasion, nodular goiter, thyroid adenoma, and Hashimoto's thyroiditis. All ultrasound imaging features and postoperative pathological features were reviewed by doctors with the title of vice-senior and above. This study was approved by the Ethics Committee of Zhejiang Provincial People's Hospital, Zhejiang Cancer Hospital, and Hangzhou First People's Hospital. All data were obtained with the patients' informed consent. The study was conducted in accordance with the Declaration of Helsinki (as revised in 2013). 

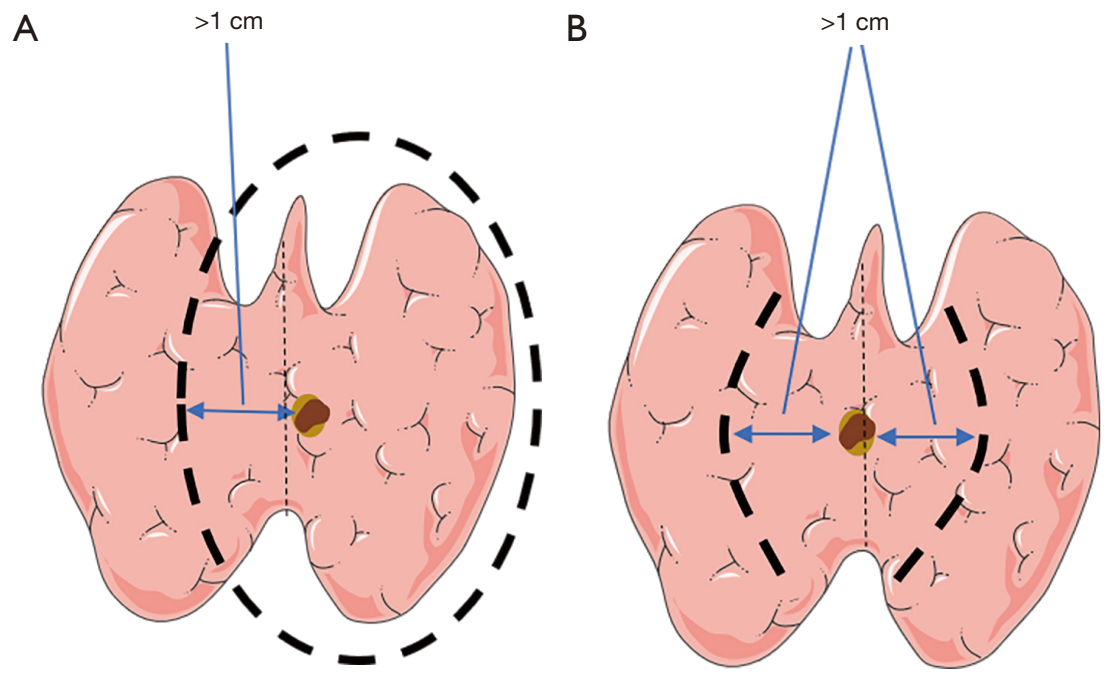

Figure 1 Schema of less-than-total thyroidectomy according to the location of the primary tumors. (A) Unilateral lobes plus isthmus combined with contralateral medial partial resection; (B) bilateral medial lobes plus isthmus resection.

\section{Preoperative assessment}

The results of preoperative color ultrasound examination of the thyroid and cervical lymph nodes of all participants were analyzed to assess tumor location, tumor size, and cervical lymph node status. The vocal cord function of participants was examined by laryngoscope, and indicators such as thyroid hormone, parathyroid hormone, calcitonin, and serum calcium were measured. The cT1N0M0 solitary PTCI in this study was defined by drawing 2 virtual lines perpendicular to the skin along the 2 edges of the trachea, with the intersection of the longest diameter and shortest diameter of the lesion considered the tumor center. If the tumor center of PTC was located between the 2 virtual lines, it was regarded as PTCI, while a solitary lesion without cervical lymph node metastasis was diagnosed as cT1N0M0 solitary PTCI. The American Joint Committee on Cancer (AJCC) thyroid cancer tumor, node, metastasis (TNM) staging system was used for tumor classification (8th edition, 2017).

\section{Surgery and follow-up}

To directly analyze the impact of different surgical methods on the prognosis of patients with cT1N0M0 solitary PTCI, participants were subdivided into groups according to the surgical method. According to the extent of thyroidectomy and lymph node dissection, 32 participants with total thyroidectomy plus unilateral central neck dissection were included in group A, and 36 participants with total thyroidectomy plus bilateral central neck dissection were included in group B. A total of 24 participants with lessthan-total thyroidectomy plus unilateral central neck dissection were included in group C, and 32 participants with less-than-total thyroidectomy plus bilateral central neck dissection were included in group D. Among them, less-than-total thyroidectomy included unilateral lobes plus isthmus combined with contralateral medial partial resection, and bilateral medial lobes plus isthmus resection. As shown in Figure 1, the range of the medial partial resection of all participants was more than $1 \mathrm{~cm}$ from the edge of the mass; and according to the final pathology report, all resection margins were negative. The surgeon decided whether to perform lateral cervical lymph node dissection based on preoperative imaging evaluation, puncture pathology report, and intraoperative exploration. If the center of the tumor deviated from the side of the midline of the isthmus, the central lymph node dissection was performed on that side; otherwise, bilateral central lymph node dissection was performed. All treatment methods were determined by consultation between the patients and their attending physician team. The operation was conducted by 3-level physicians in the group. There was no significant difference in preoperative tumor staging between different groups.

All participants were given thyroid-stimulating hormone (TSH) suppressive therapy after surgery. Routine thyroid function tests and neck B-ultrasound were performed every 
3-6 months after surgery, and neck and chest computed tomography (CT) scans were performed every 12 months. Participants suspected of recurrence were sent to the hospital for further treatment. All the recurrent cases were confirmed by pathology or imaging. Postoperative complications (patients with similar preoperative symptoms were excluded) and postoperative recurrence were investigated through case review and followup. Postoperative complications included hoarseness, hypocalcemia, choking, chylous leakage, postoperative infection, and so on. While the definition of postoperative hypocalcemia varies widely in literature $(16,17)$, the diagnostic criteria used in this study were: blood calcium level below $2.1 \mathrm{mmol} / \mathrm{L} 1$ week after total thyroidectomy, or the presentation of the following symptoms: muscle pain, muscle weakness, arrhythmia for the first time after surgery, tingling and numbness in the face and limbs, and tendon hyperreflexia; symptoms lasting more than 6 months were considered permanent hypocalcemia. The diagnostic criteria for permanent postoperative hoarseness in this study were: no improvement for more than 6 months and the presence of recurrent laryngeal nerve palsy was supported by laryngoscopy. Statistical analysis for different surgical methods was performed to understand the impact of different surgical methods on the prognosis of patients with cT1N0M0 solitary PTCI, and participant clinicalpathological characteristics were further analyzed including age, gender, tumor size, capsule invasion, co-presentation of nodular goiter, Hashimoto's thyroiditis, and adenomas, to determine their correlation with central lymph node metastasis in cT1N0M0 patients with solitary PTCI. Regression analysis was used to explore the influencing factors of the central lymph node metastasis in cT1N0M0 patients with solitary PTCI.

\section{Statistical analysis}

In statistical analysis of clinical and pathological features and postoperative complications in this study, the measurement data were represented by $\bar{x} \pm s$, and the count data were represented by the constituent ratio. The main tests used included the $t$-test, rank-sum test, and chi-square $\left(\chi^{2}\right)$ test. Recurrence-free survival was analyzed using the KaplanMeier curve (KM curve), and logistic regression analysis was used for regression analysis. All statistical analyses were performed using SPSS version 25.0 (IBM Corp., Armonk, NY, USA). A P value $<0.05$, was considered statistically significant.

\section{Results}

\section{Participant and tumor characteristics}

Participants were grouped according to the methods described in Materials and Methods, resulting in 32 (25.8\%) being assigned to group A, 36 (29\%) to group B, $24(19.4 \%)$ to group C, and $32(25.8 \%)$ to group D. Table 1 displays the participant demographic and clinicopathologic characteristics. The average sizes of primary lesions were $0.844,0.939,0.9$, and $1.063 \mathrm{~cm}$ in the 4 groups. Primary lesions ranged from $0.1-3 \mathrm{~cm}$ in diameter. Age at diagnosis in the 4 groups ranged from 21 to 70 years old. There were no significant differences between patients in age, gender, tumor size, and whether there were capsular invasion, gland dissemination accompanied with nodular goiter, adenoma, and Hashimoto's nodule $(\mathrm{P}>0.05)$.

\section{Postoperative complications}

Overall, 124 patients with CT1N0M0 solitary PTCI were followed up and the postoperative morbidity is shown in Table 2. In Group A, complications occurred in 8 participants (25.0\%), of these 2 participants experienced both complications. In Group B, complications occurred in 6 participants $(16.7 \%)$ and 1 of them experienced both complications. In Group C, complications occurred in 9 participants $(37.5 \%), 3$ of whom experienced both complications. In Group D, complications occurred in 18 participants $(56.3 \%)$ among whom 3 experienced both complications.

Statistical analysis showed that there were significant differences in the incidence of postoperative transient symptomatic hypocalcemia between the 4 groups $(\mathrm{P}=0.048)$ but yielded no significant differences in the incidence of postoperative hoarseness, choking cough when drinking water, and infection $(\mathrm{P}>0.05)$. Pairwise comparisons of the incidence of postoperative transient symptomatic hypocalcemia and a total of 6 comparisons were conducted among the 4 groups, yielding significant differences $(\mathrm{P}<0.0083)$. The incidence of symptomatic hypocalcemia was higher in group B than group D, while the differences among the remaining groups were not significant $(\mathrm{P}>0.0083)$.

\section{Postoperative recurrence}

Median follow-up was 67.5 months (27-225 months) and during the follow-up period, 5 participants experienced 
Table 1 Baseline characteristics of groups A, B, C, and D

\begin{tabular}{|c|c|c|c|c|c|c|}
\hline Characteristics & Group A, n=32 (25.8\%) & Group B, n=36 (29.0\%) & Group C, n=24 (19.4\%) & Group D, n=32 (25.8\%) & $\chi^{2}$ value & $P$ value \\
\hline \multicolumn{7}{|l|}{ Age (years) } \\
\hline Mean \pm SD & $42.09 \pm 11.02$ & $42.75 \pm 11.19$ & $44.38 \pm 9.78$ & $48.08 \pm 9.89$ & 5.163 & 0.160 \\
\hline$<55$ & $29(90.6 \%)$ & $31(86.1 \%)$ & $21(87.5 \%)$ & $26(81.3 \%)$ & 1.226 & 0.773 \\
\hline Gender & & & & & 3.653 & 0.321 \\
\hline Female & $25(78.1 \%)$ & $29(80.6 \%)$ & $17(70.8 \%)$ & $29(90.6 \%)$ & & \\
\hline Male & $7(21.9 \%)$ & $7(19.4 \%)$ & $7(29.2 \%)$ & $3(9.4 \%)$ & & \\
\hline \multicolumn{7}{|l|}{ Tumor size $(\mathrm{cm})$} \\
\hline$>0.5$ & $19(59.4 \%)$ & $20(83.3 \%)$ & $16(66.7 \%)$ & $26(81.3 \%)$ & & \\
\hline Capsular invasion & & & & & 2.068 & 0.612 \\
\hline No & $26(81.3 \%)$ & $25(69.4 \%)$ & $20(83.3 \%)$ & $25(78.1 \%)$ & & \\
\hline Yes & $6(18.8 \%)$ & $11(30.6 \%)$ & $4(16.7 \%)$ & $7(21.9 \%)$ & & \\
\hline Lobe dissemination & & & & & 2.635 & 0.450 \\
\hline No & $32(100 \%)$ & $36(100 \%)$ & $23(95.8 \%)$ & $31(96.9 \%)$ & & \\
\hline Yes & 0 & 0 & $1(4.2 \%)$ & $1(3.1 \%)$ & & \\
\hline Nodular goiter & & & & & 0.637 & 0.895 \\
\hline Hashimoto's thyroiditis & & & & & 3.503 & 0.340 \\
\hline No & $23(71.9 \%)$ & $32(88.9 \%)$ & $18(75.0 \%)$ & $24(75.0 \%)$ & & \\
\hline Yes & $9(28.1 \%)$ & $4(11.1 \%)$ & $6(25.0 \%)$ & $8(25.0 \%)$ & & \\
\hline
\end{tabular}

Group A: total thyroidectomy plus unilateral central neck dissection; Group B: total thyroidectomy plus bilateral central neck dissection; Group C: less-than-total thyroidectomy plus unilateral central neck dissection; Group D: less-than-total thyroidectomy plus bilateral central neck dissection. $\chi^{2}$, chi-square; SD, standard deviation.

local recurrences. In Group A, 3 participants (9.4\%) experienced recurrence. In Group B and D, 1 participant ( $2.8 \%, 3.1 \%$, respectively) experienced recurrence, and in Group C, there was no recurrence. Results of KM curve analysis showed that there were no significant differences in recurrence-free survival rates between the 4 groups (logrank $\mathrm{P}=0.586$ ) (Figure 2).

\section{Receiver-operating characteristic curve of the predictive model for central lymph node metastasis}

We applied receiver operating characteristic (ROC) curve analysis to evaluate the influence of the size of primary lesions on central lymph node metastasis for patients with CT1N0M0 solitary PTCI, and the results showed that 
Table 2 Comparison of postoperative complications between groups A, B, C, and D

\begin{tabular}{|c|c|c|c|c|c|c|}
\hline Variables & Group A, $n=32$ & Group B, $n=36$ & Group C, $n=24$ & Group D, $n=32$ & $\chi^{2}$ value & $P$ value \\
\hline \multicolumn{7}{|l|}{ Hypocalcemia } \\
\hline Temporary & $8(25 \%)$ & $4(11.1 \%)$ & $7(29.2 \%)$ & $13(40.6 \%)$ & 7.883 & 0.048 \\
\hline Permanent & 0 & 0 & $1(4.2 \%)$ & 0 & 3.383 & 0.194 \\
\hline Temporary & $2(6.3 \%)$ & $3(8.3 \%)$ & $3(12.5 \%)$ & 7 (21.9\%) & 4.389 & 0.244 \\
\hline Permanent & 0 & 0 & 0 & 0 & - & - \\
\hline Choking & 0 & 0 & $1(4.2 \%)$ & 0 & 3.383 & 0.194 \\
\hline Postoperative infection & 0 & 0 & 0 & $1(3.1 \%)$ & 2.808 & 0.710 \\
\hline
\end{tabular}

Group A: total thyroidectomy plus unilateral central neck dissection; Group B: total thyroidectomy plus bilateral central neck dissection; Group C: less-than-total thyroidectomy plus unilateral central neck dissection; Group D: less-than-total thyroidectomy plus bilateral central neck dissection. $\chi^{2}$, chi-square.

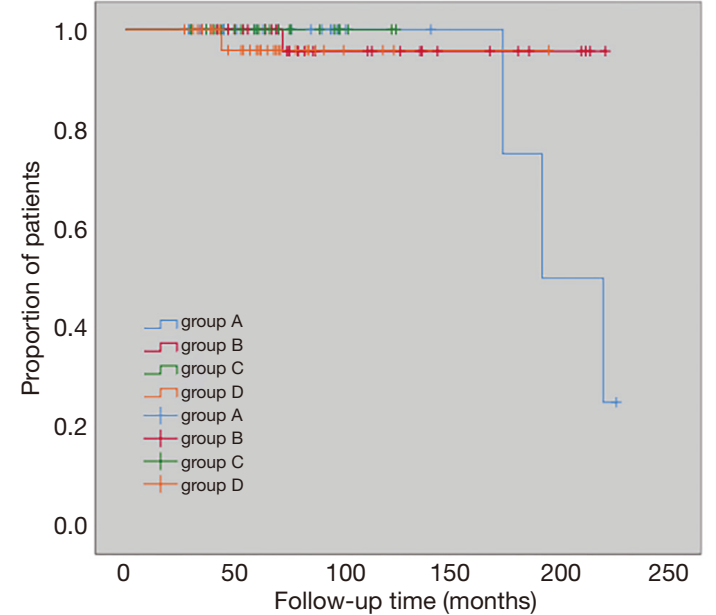

Figure 2 Association between the extent of surgery and recurrence-free survival in groups A, B, C, and D.

primary lesions $\geq 0.55 \mathrm{~cm}$ posed a higher risk for central lymph node metastasis. The area under the ROC curve (AUC) was 0.609 (95\% CI: 0.506 to $0.712, \mathrm{P}=0.047$ ), cutoff value 0.55 , sensitivity $88 \%$, specificity $33 \%$, and Youden index 0.21 (Figure 3).

\section{Multivariate logistic regression analysis}

Logistic regression analysis was performed to investigate influencing factors of central lymph node metastasis in

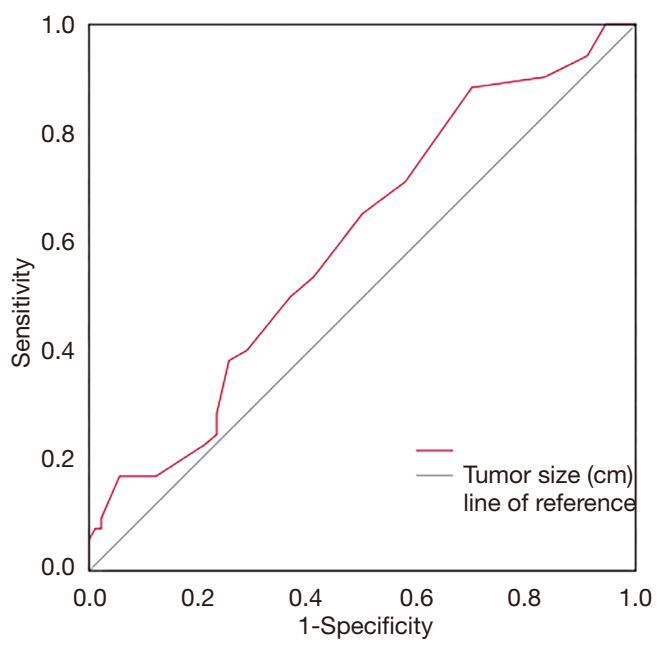

Figure 3 ROC curve for predicting central lymph node metastasis in patients with PTCI by tumor size. ROC, receiver operating characteristic; PTCI, papillary thyroid carcinoma in the isthmus.

patients with CT1N0M0 solitary PTCI (Table 3). Age under 55 years $(\mathrm{P}=0.025)$, tumor size $\geq 0.55 \mathrm{~cm}(\mathrm{P}=0.018)$, and absence of nodular goiter $(\mathrm{P}=0.04)$ were influencing factors of central lymph node metastasis in participants with CT1N0M0 solitary PTCI. There was no correlating significance between central lymph node metastasis and gender, microcarcinoma, TNM, capsular invasion, gland dissemination, adenoma, and Hashimoto's nodule $(\mathrm{P}>0.05)$. 
Table 3 Multifactorial analysis of central region lymph node metastasis in patients with cT1N0M0 PTCI with a single tumor

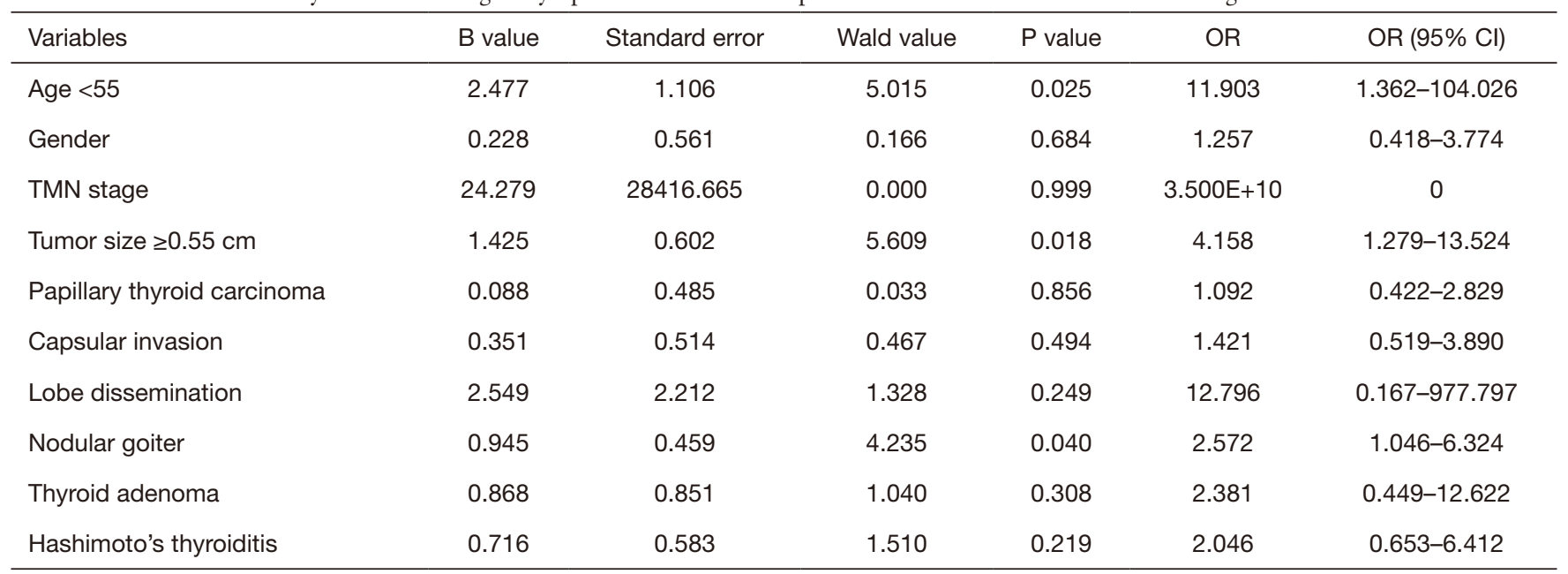

PTCl, papillary thyroid carcinoma in the isthmus; OR, odds ratio; Cl, confidence interval.

\section{Discussion}

At present, there are no clear surgical guidelines for cT1N0M0 solitary PTCI, and the focus of debate is mainly on the extent of primary tumor resection and lymph node dissection. Lim et al. (14) found that there were no significant differences in the prognosis between unilateral combined isthmus thyroidectomy and total thyroidectomy dissection for early micropapillary carcinoma with negative lymph nodes located in the thyroid isthmus. According to the follow-up data of Nixon et al. (18) and Sugenoya et al. (19), the 10- and 20-year tumor-free survival rates of patients with microscopic tumors who received isthmusectomy alone were as high as $100 \%$.

The results of this study showed that of the 124 participants with cT1N0M0 solitary PTCI, 43 (30.28\%) had capsule invasion and $83(58.45 \%)$ were complicated with nodular goiter. These values were consistent with those previously reported in the literature and significantly higher than those in patients with glandular lobe PTC (7-9,11-13). This may be due to the limited growth environment of PTCI, which makes it easier for tumor cells to spread through blood vessels or the lymphatic system, involve the capsule, and even invade extra-glandular tissues. In our study, KM analysis showed that there was no significant difference in postoperative recurrencefree survival among different extents of thyroidectomy and different methods of lymph node dissection among participants with cT1N0M0 solitary PTCI. This is in contrast to the findings of Karatzas et al. (8), who suggested that total thyroidectomy plus central neck dissection is an appropriate procedure for PTCI regardless of tumor size. In addition, patients who received total thyroidectomy plus bilateral central neck dissection were more likely to develop postoperative complications such as hypocalcemia than those who received less-than-total thyroidectomy plus bilateral central neck dissection, which was consistent with the results of Lei et al. (20). This may be because lessthan-total thyroidectomy does not touch the unilateral or bilateral superior pole of the thyroid, and can minimize injury of the parathyroid and recurrent laryngeal nerve to prevent postoperative hypocalcemia and hoarseness (21). Less-than-total thyroidectomy is more suitable for patients with cT1N0M0 solitary PTCI.

The rate of cervical lymph node metastasis in patients with PTC is $30-80 \%$ (22), which is an important factor affecting prognosis $(5,6,23)$, and the central area is the first step in cervical lymph node metastasis. Related studies have shown that tumor size is closely related to central lymph node metastasis (24-26). Using the existing data of patients with cT1N0M0 solitary PTCI, we performed ROC curve analysis (Figure 3) between tumor size and central lymph node metastasis. The AUC value was 0.609 , cutoff value 0.55 , sensitivity $88 \%$, and specificity $30 \%$. This showed that the model has a certain function in predicting central lymph node metastasis, and a screening index was obtained; that is, cT1N0M0 solitary PTCI patients with primary tumors $\geq 0.55 \mathrm{~cm}$ are prone to central lymph node metastasis. Several studies have shown that the younger the patient, the higher the rate of lymph node metastasis in the 
central region of the neck (26-28). In the 8th edition of the TNM staging of thyroid cancer of the AJCC (29), the age cut-off point was raised to 55 years, which was also used as the cut-off point for this study. Logistics multivariate analysis showed that tumor size $\geq 0.55 \mathrm{~cm}$, age $<55$ years old, and no nodular goiter were the influencing factors of central lymph node metastasis in patients with cT1N0M0 solitary PTCI. Our findings revealed that nodular goiter associated with PTCI was a protective factor for lymph node metastasis in patients with PTC, which is consistent with those of previous studies (30). Therefore, we believe that this kind of patient should actively perform central lymph node dissection. Because the thyroid isthmus is located in the center of the thyroid gland, it is difficult to determine on which side the patient's central area is located. Therefore, whether bilateral central neck dissection should be performed remains controversial. Related studies have shown that the rate of lymph node metastasis in the bilateral central region of PTCI patients was significantly higher than that of the unilateral glandular lobe PTC patients $(10,11,31)$. It has also been reported that there is a higher metastatic rate for prelaryngeal lymph nodes and contralateral paratracheal lymph nodes around the thyroid gland in patients with PTCI $(32,33)$. It may because the isthmus is located in the center of the gland, which connects the lymphatic vessels of the bilateral glandular lobes and is more likely to have bilateral lymph node metastasis (11). In this study, bilateral central lymph node metastasis occurred in 5 of the 124 participants with cT1N0M0 solitary PTCI, indicating the possibility of bilateral central lymph node metastasis in such patients. At present, some academics remain cautious about the choice of bilateral central neck dissection. The main reason is that the extent of such an operation may cause tissue injury, which may damage the recurrent laryngeal nerve, leading to hoarseness, dyspnea, and asphyxia, and potentially seriously affect the quality of life of patients after operation, even endanger their lives (34). Our data showed that there was no significant difference in the incidence of postoperative complications between participants who received bilateral central neck dissection and those who received unilateral central neck dissection. This may be related to the application of intraoperative lymph node contrast agents and nerve monitoring techniques $(35,36)$. Therefore, we believe that postoperative complications should not be used as the main reference factor for bilateral central neck dissection.

Although we analyzed cases at 3 healthcare centers, a few possible limitations still applied to this study. A relatively small sample size, short follow-up time, lower incidence rate of cT1N0M0 solitary PTC, and differences in skill level of surgeons at different healthcare centers may have led to the presence of bias. The retrospective nature of our study may also have led to selective bias. To overcome these limitations, follow-up time and the number of healthcare centers involved should be extended.

\section{Conclusions}

In conclusion, PTCI is more likely to be associated with pathological features such as multifocality and invasion of capsular and surrounding tissues, but there was no significant difference in various surgical modalities for recurrence and metastasis of cT1N0M0 solitary PTCI. Patients are more likely to develop postoperative complications such as hypocalcemia and hoarseness after total thyroidectomy; thus, patients with cT1N0M0 solitary PTCI are recommended to undergo less-than-total thyroidectomy. Among such patients, central lymph node dissection was recommended for those who were prone to have central occult lymph node metastases with tumor size $\geq 55 \mathrm{~cm}$, age under 55 years, and without nodular goiter.

\section{Acknowledgments}

We are grateful to Zhejiang Provincial People's Hospital, Cancer Hospital of The University of Chinese Academy of Sciences, and Hangzhou First People's Hospital for their support of this study.

Funding: This work was supported by grants from the Key Research and Development Program of Zhejiang Province (2021C0308), the National Natural Science Foundation of China (81802674), National Natural Science Foundation of China-Zhejiang Joint Fund (U20A20382), Zhejiang Provincial Natural Science Foundation of China (LY21H160049), the Medical and Health Research Program of Zhejiang Province (2021419088), and Zhejiang TCM Excellence Young Talents Fund Project (2018ZQ010).

\section{Footnote}

Reporting Checklist: The authors have completed the STARD reporting checklist. Available at https://dx.doi. org/10.21037/gs-21-357

Data Sharing Statement: Available at https://dx.doi. org/10.21037/gs-21-357 
Conflicts of Interest: All authors have completed the ICMJE uniform disclosure form (available at https://dx.doi. org/10.21037/gs-21-357). The authors have no conflicts of interest to declare.

Ethical Statement: The authors are accountable for all aspects of the work in ensuring that questions related to the accuracy or integrity of any part of the work are appropriately investigated and resolved. The study was conducted in accordance with the Declaration of Helsinki (as revised in 2013). This study was approved by the Ethics Committee of Zhejiang Provincial People's Hospital, Zhejiang Cancer Hospital, and Hangzhou First People's Hospital. All data were obtained with the patients' informed consent.

Open Access Statement: This is an Open Access article distributed in accordance with the Creative Commons Attribution-NonCommercial-NoDerivs 4.0 International License (CC BY-NC-ND 4.0), which permits the noncommercial replication and distribution of the article with the strict proviso that no changes or edits are made and the original work is properly cited (including links to both the formal publication through the relevant DOI and the license). See: https://creativecommons.org/licenses/by-nc-nd/4.0/.

\section{References}

1. Sung H, Ferlay J, Siegel RL, et al. Global Cancer Statistics 2020: GLOBOCAN Estimates of Incidence and Mortality Worldwide for 36 Cancers in 185 Countries. CA Cancer J Clin 2021;71:209-49.

2. Lim H, Devesa SS, Sosa JA, et al. Trends in Thyroid Cancer Incidence and Mortality in the United States, 1974-2013. JAMA 2017;317:1338-48.

3. Wiltshire JJ, Drake TM, Uttley L, et al. Systematic Review of Trends in the Incidence Rates of Thyroid Cancer. Thyroid 2016;26:1541-52.

4. Cecoli F, Ceresola EM, Altrinetti V, et al. Therapeutic Strategies and Clinical Outcome in Papillary Thyroid Microcarcinoma: A Multicenter Observational Study. Eur Thyroid J 2016;5:180-6.

5. Haugen BR, Alexander EK, Bible KC, et al. 2015 American Thyroid Association Management Guidelines for Adult Patients with Thyroid Nodules and Differentiated Thyroid Cancer: The American Thyroid Association Guidelines Task Force on Thyroid Nodules and Differentiated Thyroid Cancer. Thyroid 2016;26:1-133.
6. Perros P, Boelaert K, Colley S, et al. Guidelines for the management of thyroid cancer. Clin Endocrinol (Oxf) 2014;81 Suppl 1:1-122.

7. Lee YS, Jeong JJ, Nam KH, et al. Papillary carcinoma located in the thyroid isthmus. World J Surg 2010;34:36-9.

8. Karatzas T, Charitoudis G, Vasileiadis D, et al. Surgical treatment for dominant malignant nodules of the isthmus of the thyroid gland: A case control study. Int J Surg 2015;18:64-8.

9. Lee YC, Na SY, Chung H, et al. Clinicopathologic characteristics and pattern of central lymph node metastasis in papillary thyroid cancer located in the isthmus. Laryngoscope 2016;126:2419-21.

10. Song CM, Lee DW, Ji YB, et al. Frequency and pattern of central lymph node metastasis in papillary carcinoma of the thyroid isthmus. Head Neck 2016;38 Suppl 1:E412-6.

11. Hahn SY, Han BK, Ko EY, et al. Ultrasound findings of papillary thyroid carcinoma originating in the isthmus: comparison with lobe-originating papillary thyroid carcinoma. AJR Am J Roentgenol 2014;203:637-42.

12. Chai YJ, Kim SJ, Choi JY, et al. Papillary thyroid carcinoma located in the isthmus or upper third is associated with Delphian lymph node metastasis. World J Surg 2014;38:1306-11.

13. Goldfarb M, Rodgers SS, Lew JI. Appropriate surgical procedure for dominant thyroid nodules of the isthmus 1 cm or larger. Arch Surg 2012;147:881-4.

14. Lim ST, Jeon YW, Suh YJ. Correlation Between Surgical Extent and Prognosis in Node-Negative, Early-Stage Papillary Thyroid Carcinoma Originating in the Isthmus. World J Surg 2016;40:344-9.

15. Song Q, Gao H, Tian X, et al. Evaluation of UltrasoundGuided Radiofrequency Ablation as a Treatment Option for Papillary Thyroid Microcarcinoma in the Isthmus: A Retrospective Study. Front Endocrinol (Lausanne) 2021;11:599471.

16. Stack BC Jr, Bimston DN, Bodenner DL, et al. American Association of Clinical Endocrinologists and American College of Endocrinology Disease State clinical review: postoperative hypoparathyroidism--definitions and management. Endocr Pract 2015;21:674-85.

17. Kazaure HS, Zambeli-Ljepovic A, Oyekunle T, et al. Severe Hypocalcemia After Thyroidectomy: An Analysis of 7366 Patients. Ann Surg 2019. [Epub ahead of print]. doi: 10.1097/SLA.0000000000003725.

18. Nixon IJ, Palmer FL, Whitcher MM, et al. Thyroid isthmusectomy for well-differentiated thyroid cancer. Ann Surg Oncol 2011;18:767-70. 
19. Sugenoya A, Shingu K, Kobayashi S, et al. Surgical strategies for differentiated carcinoma of the thyroid isthmus. Head Neck 1993;15:158-60.

20. Lei J, Zhu J, Li Z, et al. Surgical procedures for papillary thyroid carcinoma located in the thyroid isthmus: an intention-to-treat analysis. Onco Targets Ther 2016;9:5209-16.

21. Skilbeck C, Leslie A, Simo R. Thyroid isthmusectomy: a critical appraisal. J Laryngol Otol 2007;121:986-9.

22. Dimov RS. The effect of neck dissection on quality of life in patients with differentiated thyroid cancer. Gland Surg 2013;2:219-26.

23. Smith VA, Sessions RB, Lentsch EJ. Cervical lymph node metastasis and papillary thyroid carcinoma: does the compartment involved affect survival? Experience from the SEER database. J Surg Oncol 2012;106:357-62.

24. Zhao Q, Ming J, Liu C, et al. Multifocality and total tumor diameter predict central neck lymph node metastases in papillary thyroid microcarcinoma. Ann Surg Oncol 2013;20:746-52.

25. Wu Q, Li Y, Wang Y, et al. Sonographic features of primary tumor as independent predictive factors for lymph node metastasis in papillary thyroid carcinoma. Clin Transl Oncol 2015;17:830-4.

26. Zheng X, Peng C, Gao M, et al. Risk factors for cervical lymph node metastasis in papillary thyroid microcarcinoma: a study of 1,587 patients. Cancer Biol Med 2019;16:121-30.

27. Malandrino P, Pellegriti G, Attard M, et al. Papillary thyroid microcarcinomas: a comparative study of the characteristics and risk factors at presentation in two cancer registries. J Clin Endocrinol Metab 2013;98:1427-34.

28. Ito $\mathrm{Y}$, Miyauchi A, Kihara M, et al. Patient age is significantly related to the progression of papillary

Cite this article as: Zhang $\mathrm{LZ}, \mathrm{Xu} J \mathrm{~J}$, Ge XY, Wang KJ, Tan Z, Jin TF, Zhang WC, Li QL, Luo DC, Ge MH. Pathological analysis and surgical modalities selection of cT1N0M0 solitary papillary thyroid carcinoma in the isthmus. Gland Surg 2021;10(8):2445-2454. doi: 10.21037/gs-21-357 microcarcinoma of the thyroid under observation. Thyroid 2014;24:27-34.

29. Shaha AR, Migliacci JC, Nixon IJ, et al. Stage migration with the new American Joint Committee on Cancer (AJCC) staging system (8th edition) for differentiated thyroid cancer. Surgery 2019;165:6-11.

30. Ma H, Li L, Li K, et al. Hashimoto's thyroiditis, nodular goiter or follicular adenoma combined with papillary thyroid carcinoma play protective role in patients. Neoplasma 2018;65:436-40.

31. Chang YW, Lee HY, Kim HS, et al. Extent of central lymph node dissection for papillary thyroid carcinoma in the isthmus. Ann Surg Treat Res 2018;94:229-34.

32. Seok J, Choi JY, Yu HW, et al. Papillary Thyroid Cancers of the Thyroid Isthmus: The Pattern of Nodal Metastasis and the Significance of Extrathyroidal Extension. Ann Surg Oncol 2020;27:1937-44.

33. Liu N, Yang Y, Chen B, et al. The Extent of Therapeutic Central Compartment Neck Dissection in Unilateral cT1N1a or cT2N1a Papillary Thyroid Carcinoma. Cancer Manag Res 2020;12:12801-9.

34. Rosenbaum MA, McHenry CR. Central neck dissection for papillary thyroid cancer. Arch Otolaryngol Head Neck Surg 2009;135:1092-7.

35. Wang B, Qiu NC, Zhang W, et al. The role of carbon nanoparticles in identifying lymph nodes and preserving parathyroid in total endoscopic surgery of thyroid carcinoma. Surg Endosc 2015;29:2914-20.

36. Li Y, Jian WH, Guo ZM, et al. A Meta-analysis of Carbon Nanoparticles for Identifying Lymph Nodes and Protecting Parathyroid Glands during Surgery. Otolaryngol Head Neck Surg 2015;152:1007-16.

(English Language Editor: J. Jones) 